\title{
Deafening-Induced Vocal Deterioration in Adult Songbirds Is Reversed by Disrupting a Basal Ganglia-Forebrain Circuit
}

\author{
K. W. Nordeen and E. J. Nordeen \\ Department of Brain and Cognitive Sciences, University of Rochester, Rochester, New York 14627
}

\begin{abstract}
Motor exploration can be an adaptive strategy when behavior fails to achieve an expected outcome. For example, like humans, adult songbirds change their vocal output when auditory feedback is altered or absent. Here, we show that the output of an anterior forebrain pathway (AFP) through the avian basal ganglia directly contributes to the expression of deafening-induced vocal changes in adulthood. Lesioning the output nucleus of this circuit in adult male zebra finches reverses moderate changes in song structure and variability caused by deafening. Furthermore, the results indicate that more severe deafening-induced changes in vocal behavior likely reflect altered function outside the AFP (e.g., within the vocal motor pathway). AFP lesions do not promote recovery if songs are severely deteriorated at the time of lesion even though previous work shows that the AFP is required for such deterioration to emerge. Thus, in birds, as in mammals, the contribution of basal ganglia-thalamic-cortical circuits to motor control may change when feedback is absent or unexpected and includes both "active" and "permissive" roles.
\end{abstract}

\section{Introduction}

When behavior fails to produce an expected outcome, cognitivemotor systems detect this discrepancy and promote behavioral change. Avian song learning and maintenance illustrate this process. During learning, birds explore vocal motor patterns and retain those matching a previously stored auditory template (Marler and Peters, 1982; Tchernichovski et al., 2001). In adulthood, birds adjust their song adaptively in response to specific distortions of auditory feedback and readjust vocal output when normal feedback is restored (Andalman and Fee, 2009; Sober and Brainard, 2009). Adult songbirds also change their song after deafening (Nordeen and Nordeen, 1992; Okanoya and Yamaguchi, 1997), reminiscent of the gradual deterioration of human speech after postlingual deafening (Waldenstein, 1990; Cowie and Douglas-Cowie, 1992). Together, these data suggest that adult vocal plasticity may engage the same mechanisms used during early vocal learning.

Here, we investigate how a basal ganglia-thalamic-pallial circuit contributes to deafening-induced vocal degradation in songbirds. We focus on the output of this circuit, the lateral magnocellular nucleus of the anterior nidopallium (LMAN). LMAN is a forebrain region (functionally akin to mammalian prefrontal cortex) that projects directly to the robust nucleus of the archopallium (RA), a component of the descending vocal motor pathway (VMP). HVC, an upstream component of the VMP, also projects to RA. Although LMAN lesions do

\footnotetext{
Received Dec. 14, 2009; revised April 5, 2010; accepted April 13, 2010.

This work was supported by grants from the National Science Foundation (IOS-0814219) and from the Kilian J. and Caroline F. Schmitt Foundation. We thank Heather Bradstreet, Diamond Ling, and Maryalice Wolfe for technica support.

Correspondence should be addressed to Ernest J. Nordeen, Department of Brain and Cognitive Sciences, University of Rochester, Rochester, NY 14627. E-mail: ernie@bcs.rochester.edu.

DOI:10.1523/JNEUROSCI.6181-09.2010

Copyright $\odot 2010$ the authors $\quad 0270-6474 / 10 / 307392-09 \$ 15.00 / 0$
}

not disrupt stable song behavior in adults, lesions made before auditory feedback is altered or eliminated prevent the vocal changes that normally occur (Williams and Mehta, 1999; Brainard and Doupe, 2000b). Furthermore, lesioning LMAN during song development arrests sensorimotor learning by promoting premature stabilization of vocal output (Bottjer et al., 1984).

While current data suggest that LMAN is critical for feedbackdriven vocal plasticity even in adult songbirds, it remains unclear what role(s) it plays in facilitating vocal change when auditory feedback is unavailable. LMAN could contribute directly to the expression of vocal changes seen after deafening since stimulating LMAN triggers immediate changes in song structure (Kao et al., 2005), and this region contributes to natural song variability in both young and adult birds (Kao et al., 2005; Olveczky et al., 2005; Kao and Brainard, 2006; Aronov et al., 2008). Also, LMAN lesions eliminate recently learned adjustments in song provoked by aversive feedback (Andalman and Fee, 2009) and rapidly reverse vocal change produced by microlesions of the descending VMP (Thompson et al., 2007). LMAN may also prompt or permit changes elsewhere. For instance, LMAN delivers brain-derived neurotrophic factor (BDNF) to the VMP (Johnson et al., 1997), and this could foster vocal change after deafening (Kittelberger and Mooney, 2005).

We report here that lesioning LMAN after deafeninginduced alterations in song behavior first emerge produces a rapid return toward the predeaf song pattern, indicating that LMAN actively degrades vocal output after deafening. However, LMAN lesions do not restore profoundly deteriorated songs, suggesting that severe vocal changes, while dependent on LMAN for their initiation (Brainard and Doupe, 2000b), reflect changes in other parts of the song system. Thus, LMAN actively alters song after deafening but also permits changes within other song system circuits. 


\section{Materials and Methods}

The University of Rochester's Institutional Animal Care and Use Committee (Rochester, New York) approved all procedures used in this study. Zebra finches (Taeniopygia guttata) were bred and raised in our laboratory on a 14/10 h light/dark cycle with food and seed available ad libitum. Juveniles were reared by their parents and segregated $65 \mathrm{~d}$ after hatching into single-sex flight aviaries.

Surgery and sound recording. Beginning at 90-100 d posthatch, songs produced in the presence of a female were recorded weekly with Shure 16A microphones and digitized using Avisoft SASLab Pro software. Adult zebra finch song is usually initiated by repetitions of a single introductory syllable followed by several repetitions of a motif consisting of 2-8 unique syllables organized into a stable sequence. Spectrographs of songs were visually evaluated to determine whether syllable and motif structure were stable across at least 2 weeks. Birds producing a stable song were bilaterally deafened by cochlear extirpation immediately after a final baseline recording $(\mathrm{d} 0)$. The average age at deafening was $132 \mathrm{~d}$ (range, 122-146). Procedures for deafening were identical to those used previously in our laboratory (Nordeen and Nordeen, 1992; Scott et al., 2007), and extracted cochlea were examined under a microscope to confirm that the distal end (lagena) was intact. Songs were recorded again within $3 \mathrm{~d}$ after deafening and weekly thereafter to monitor changes in song structure.

The time course of deafening-induced vocal deterioration among young adult zebra finches can vary considerably (Nordeen and Nordeen, 1992; Brainard and Doupe, 2000b, 2001; Horita et al., 2008), with some birds exhibiting only minor changes even months after deafening. This variability made it possible to assess how LMAN lesions would affect behavior in relation to both the severity of vocal degradation and the time elapsed after deafening. When reliable changes in song structure were detected after deafening, some of the birds were anesthetized with isoflurane and LMAN was lesioned bilaterally ( $100 \mu \mathrm{A}$ for $35-45 \mathrm{~s}$ at each of 4-6 sites/hemisphere). The remaining birds survived an additional 8 weeks before lesioning LMAN. Songs were rerecorded on the morning of LMAN lesion (L) and on postlesion days 1, 3, 7, 10, 14, 28, 48, and 84 .

Verification of LMAN lesions. After the last song recording, birds were perfused $(0.9 \%$ saline followed by $4 \%$ paraformaldehyde) and their brains were frozen and then sectioned $(30 \mu \mathrm{m})$ and stained with thionine to evaluate the completeness of LMAN ablation. To establish the average volume of LMAN in control tissue, six age-matched controls were processed similarly for histological analysis. In each animal, cross-sectional areas of left and right LMANs were measured every $90 \mu \mathrm{m}$ using NIH Image software. The volume of the nucleus then was estimated using the formula for a cone frustrum (Tramontin et al., 1998). Lesion size is expressed as percentage of mean control LMAN volume (left plus right).

Selection of songs for analysis. After discarding the first 20 songs of each recording day, we selected for analysis the next 20 song files that were free of contamination by cage noise or female call notes. For each bird, 20 motif files (the initial motif and a single preceding introductory note, if present) were created from the recordings obtained $1-2 \mathrm{~h}$ before deafening ( $\mathrm{d} 0$ ). These $\mathrm{d} 0$ motifs were used as templates against which we compared songs produced at other times. The initial motif of each song bout was selected for two reasons: 1) terminal motifs can be truncated and thus do not always include all song syllables that comprise the canonical motif; and 2) the number of motifs/bouts tend to decline after deafening, and many deafened birds eventually produced song bouts that consisted of only a single motif.

For birds that retained their motif structure after deafening, 20 motif files were generated as described above for each subsequent recording day, and these were compared with the $\mathrm{d} 0$ motif templates. For birds that failed to retain their song motifs after deafening, we obtained 20 long samples of song produced on a given recording day. These long samples began with an introductory note (if present), included all syllables produced in that song bout, and included multiple renditions of most or all song syllables. Thus, this analysis was less stringent than the motif analysis because there were multiple opportunities for a given song syllable to match the $\mathrm{d} 0$ templates. To ensure that the pattern of results revealed by the two different methods was similar, we also conducted this long sample analysis in two animals that retained motif structure (see Results).

Measurement of deafening and lesion-induced changes in overall song structure. We used Sound Analysis Pro (SA+) (Tchernichovski et al., $2000,2001)$ to assess how deafening and subsequent LMAN lesions affected overall motif structure. For this analysis, motif files obtained on d0 were used as the "template" for each bird, and songs recorded on other days were evaluated with respect to these do samples. The similarity between songs produced on different days was measured using the SA+ asymmetric similarity analysis that calculates similarity based on measurements of pitch, frequency modulation (FM), amplitude modulation, Wiener entropy (noisiness), and goodness of pitch (how periodic or harmonic the acoustic structure is). Three separate measures of song similarity were obtained from SA + as follows: 1) percentage similarity (the percentage of the template ( $\mathrm{d} 0$ motif) achieving a threshold level of similarity to the sample motif); 2) accuracy (the average local similarity score for those sections of the sample motif that are judged to be similar to the template); and 3) sequential match (the degree to which similar sounds in the sample and template correspond in temporal order). From these scores a fourth measure, global similarity (GS), was also calculated. In birds that retained their motif, this global score reflected all three measures $\left(\mathrm{GS}_{1}=\right.$ percentage similarity $*$ accuracy $*$ sequential match $)$. For birds that lost motif structure, the presence of multiple renditions of syllables in the long song sample renders the sequential match score uninformative. Thus, for the long sample analysis, the global score reflected only two similarity measures $\left(\mathrm{GS}_{2}=\right.$ percentage similarity $*$ accuracy). Preliminary analysis confirmed that a sample size of 15-20 song renditions was sufficient to achieve stable scores.

The various measures of similarity were used to evaluate the effects of deafening and LMAN lesions both within individual birds and across birds (group analysis). Each of the 20 sample motifs (or long samples) produced by a bird on a given day was assigned average scores based on comparisons to each of the 20 template (d0) motifs. Thus, each bird had 20 estimates of each similarity score for each of the following days sampled: 1-2 weeks before deafening (d-7), weekly after deafening (d7, d14, $\mathrm{d} 21$, etc), the morning of LMAN lesion (L0), and 1, 3, 7, 14, 28, 48, $84 \mathrm{~d}$ after LMAN lesion (L1, L3, L7, etc). For a given dependent measure, within-bird analyses used the 20 scores generated for each recording day, while the group analyses used the overall mean calculated for each bird on each recording day. We also calculated the coefficient of variation (CV; SD/mean of 20 samples) to estimate how variable the birds' motifs were on a given day with respect to their similarity to the battery of $\mathrm{d} 0$ "template" motifs.

To obtain a benchmark for song similarity scores, we compared predeaf song motifs $(n=20)$ produced by each bird to predeaf motifs produced by unrelated males. Similarly, we compared long samples of predeaf song to predeaf motifs from unrelated males to obtain a benchmark for the long sample analysis that was required for birds that lost motif structure after deafening.

As noted, song degradation after deafening varied across birds. Thus, to evaluate which proportion of the deafening-induced decline in song similarity scores could be rescued by LMAN lesions for each bird, we calculated the severity of deafening-induced degradation and the extent of lesion-induced recovery as follows: percentage deterioration at $\mathrm{L} 0=$ $\left(G S_{\mathrm{d}-7}-G S_{\mathrm{L} 0}\right) / G S_{\mathrm{d}-7} ;$ percentage deterioration at $\mathrm{L} 7=\left(G S_{\mathrm{d}-7}-G S_{\mathrm{L} 7}\right) /$ $G S_{\mathrm{d}-7}$; percentage recovery at $\mathrm{L} 7=1-[$ (percentage deterioration at $\mathrm{L} 7) /\left(\right.$ percentage deterioration at L0)]; where $G S_{\mathrm{d}-7}, G S_{\mathrm{L} 0}$, and $G S_{\mathrm{L} 7}$ are GS scores for d-7, L0, and L7, respectively.

Adult deafening promotes a decline in the number of motifs per song bout (Horita et al., 2008), and so we also explored whether LMAN lesions affect this aspect of song structure. We counted the number of motifs produced in each of 20 complete song bouts produced on $\mathrm{d} 0$, L0, and L7 and calculated a daily average number of motifs per bout.

Statistical analyses of deafening- and lesion-induced changes in overall song structure. Statistical analyses of group data used nonparametric statistics because deafening and LMAN lesions produced significant group differences in variance. All effects were evaluated using two-tailed tests with significance level set at $p \leq 0.05$. Effects of deafening on song similarity scores across all animals were assessed by Wilcoxon paired $t$ tests 
(Bonferonni adjusted), comparing scores on d-7 to those obtained $14 \mathrm{~d}$ after deafening (d14) and those obtained just before LMAN lesion (L0). Within each bird, deafeninginduced changes in similarity scores were assessed using unpaired $t$ tests comparing $\mathrm{d}-7$ to L0; Welch's correction was applied where appropriate to correct for nonhomogeneity of variance. Effects of LMAN lesions on song similarity scores across all animals were evaluated by Friedman repeated-measures ANOVA followed by Bonferonni adjusted pairwise comparisons. Within individual birds, lesion effects were assessed using unpaired $t$ tests coupled with Welch's correction where appropriate.

Deafening-induced changes in the CV of similarity scores were assessed by paired $t$ tests, and lesion effects were assessed by Friedman repeated-measures ANOVA followed by Bonferonni adjusted pairwise comparisons. The proportion of animals exhibiting improvement in similarity scores during the week prelesion versus the week postlesion was evaluated by a $\chi^{2}$ test. Finally, overall effects of deafening and LMAN lesions on the number of motifs per bout were evaluated by paired $t$ tests comparing $\mathrm{d} 0$ versus $\mathrm{L} 0$ and $\mathrm{L} 0$ versus L7. Effects in individual birds were assessed using unpaired $t$ tests comparing d0 to L0 and L0 to L7, coupled with Welch's correction where appropriate.

Measurement and statistical analysis of deafening-and lesion-induced changes in syllable structure and variability. To understand how changes in the structure of individual syllables contributed to changes in motif similarity, we used the features batch module of SA+ to measure the acoustic character and consistency of identified song syllables at d0, L0, and L7. Because severe syllable deterioration sometimes precluded our ability to unequivocally identify d0 syllables in L0 and L7 songs, this feature analysis necessarily focused upon syllables that were only moderately deteriorated by the time of LMAN lesion. The analysis included all animals in which at least $50 \%$ of predeaf syllables could be positively identified at each of the three time points ( 53 syllables drawn from 11 birds; $3-7$ syllables/bird). One goal was to establish how deafening and LMAN lesions affected specific acoustic features of these syllables. Thus, for each syllable we measured syllable duration, mean pitch, mean FM, mean entropy, mean pitch goodness, and mean frequency. Also, to capture how much these features were modulated within each syllable, we measured the variation in mean pitch, FM, entropy, pitch goodness, and mean frequency (fast Fourier transform window, $9.27 \mathrm{~ms}$; advance, $1.36 \mathrm{~ms}$ ). Finally, given the contribution of LMAN to how consistent these 11 acoustic features of a syllable are across renditions (Kao et al., 2005; Olveczky et al., 2005; Kao and Brainard, 2006; Hampton et al., 2009) we measured the coefficient of variation $(\mathrm{CV}=\mathrm{SD} /$ mean $)$ of each of these measures.

For each recording day, a WAV (waveform audio file format) file was created for each syllable that consisted of a minimum of 20 exemplars drawn from 20 different song bouts. For each syllable, SA+ settings for amplitude, entropy, and minimum syllable and gap duration were adjusted to reliably outline do exemplars, and these settings were then applied to the L0 and L7 files. For each syllable, an average value for each feature and its variance throughout the syllable was calculated for each day, and the $\mathrm{CV}$ of these measures also was calculated. Wilcoxon paired $t$ tests (two-tailed) were used to assess effects of deafening and LMAN lesions on these measures, comparing d0 to L0 and L0 to L7.

\section{Results}

\section{Deafening-induced deterioration of motif structure} and consistency

All 15 birds used in the present study exhibited significant vocal deterioration after deafening. Although all were deafened between 122 and $146 \mathrm{~d}$ posthatch, the time course of vocal change varied considerably (Fig. 1). By 2 weeks after deafening, several birds produced songs that did not obviously differ from their baseline (predeaf) song, many others had begun to drop syllables or produced syllables that clearly deviated in structure from predeaf song, while the songs of three birds had deteriorated so severely that a motif structure was no longer evident. Group-level analysis of the 12 birds that continued to produce structured motifs at 2 weeks after deafening confirmed that their global similarity $\mathrm{GS}_{1}$ score (percentage similarity * accuracy $*$ sequential match) was significantly lower 2 weeks after deafening than before deafening $(\mathrm{d}-7=75.9 \pm 1.3, \mathrm{~d} 14=54.4 \pm 4.3 ; W=78$, $p=0.001$, where $W$ is the Wilcoxon test statistic). Deafening also significantly increased the variance of this $\mathrm{GS}_{1}$ score [d-7 vs d14; $\left.F_{(11,11)}=10.57 ; p=0.0002\right]$, further reflecting individual differences in the time course of deafening-induced vocal deterioration. Neither the baseline (d-7) $\mathrm{GS}_{1}$ score nor age at deafening correlated with the degree of vocal deterioration by 2 weeks after deafening.

By the morning of LMAN lesion (L0; 12-90 d postdeaf), nine birds still retained a recognizable motif structure. Group analysis of these birds revealed that $\mathrm{GS}_{1}$ scores on L0 were significantly lower than predeaf values ( $W=45, p<0.005$ ) (Fig. 2, left axis). In fact, by L0 this score had declined significantly in each of these subjects individually ( $t \geq 3.6 ; p<0.002)$. Moreover, group analyses of each measure comprising the $\mathrm{GS}_{1}$ score (\% similarity, accuracy, and sequential match) confirmed that by L0, each had declined significantly from predeaf values $(W=45, p<0.005)$ (Fig. 2, right axis). In those birds that did not retain motif structure after deafening $(n=6)$, the alternate "long sample analysis" (see Materials and Methods) confirmed a similar effect of deafening. Group analysis of these birds revealed that $\mathrm{GS}_{2}$ scores (percentage similarity $*$ accuracy) on L0 were significantly lower than predeaf values ( $W=21, p<0.05$ ) (Fig. 3 ), and each bird individually exhibited a significant decline in $\mathrm{GS}_{2}$ from predeaf to $\mathrm{L} 0$ $(t \geq 11.2, p<0.0001)$. Separate group analyses of the percentage similarity and accuracy scores for this subgroup of animals confirmed that by L0, deafening had significantly reduced both of these measures ( $W \geq 21, p<0.05$ ) (Fig. 3).

\section{LMAN lesion-induced recovery of motif structure}

LMAN lesions rapidly reversed moderate but not severe deafeninginduced changes in song similarity scores. Most lesions encompassed the caudal portion of LMAN where RA-projecting axons leave the nucleus, but some portion of LMAN was spared 


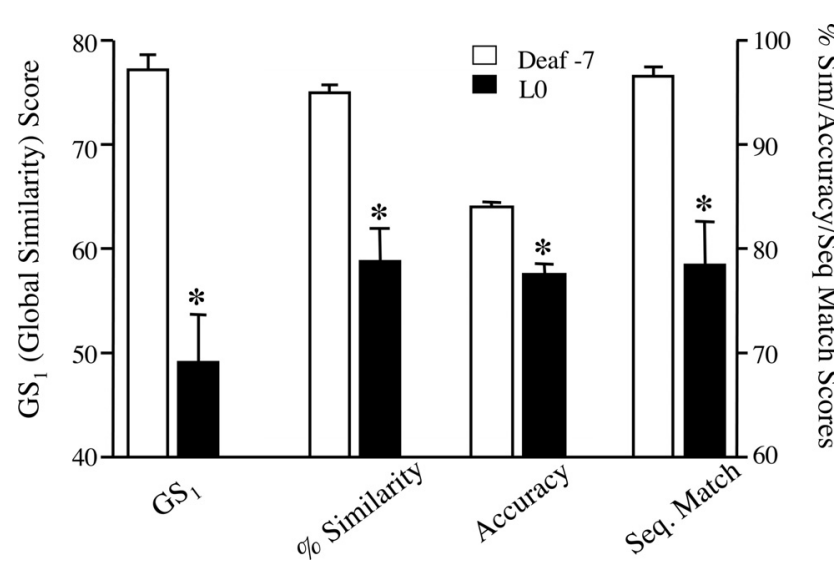

Figure 2. Deafening-induced decline in song similarity scores for birds that maintained a motif structure throughout the experiment $(n=9)$. By the day of LMAN lesions, the global similarity $\mathrm{GS}_{1}$ (left axis), as well as each of the component scores [percentage similarity, accuracy, and sequential match (Seq. Match); right axis], were significantly lower than their predeaf baseline. Data shown are group means \pm SEM, ${ }^{*} p<0.005$ (deaf-7 vs L0).

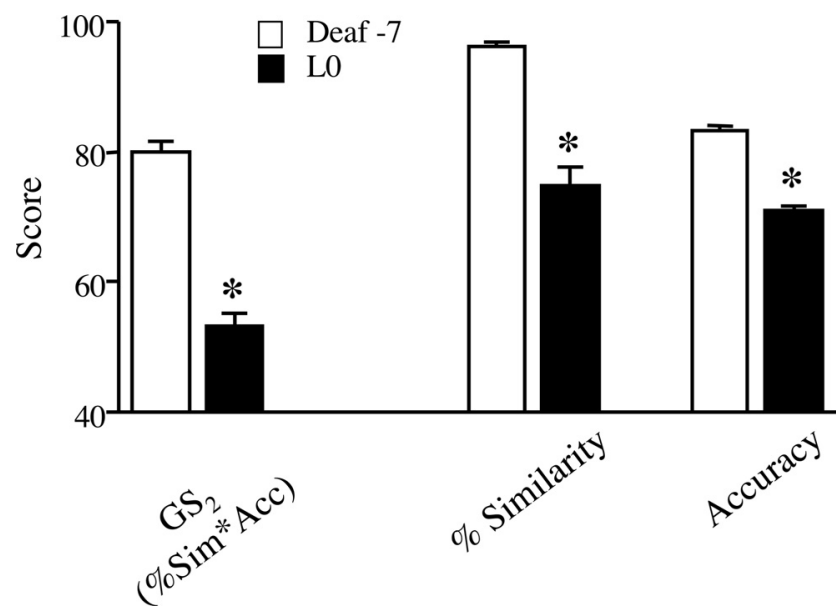

Figure 3. Song similarity scores for birds that did not maintain a motif structure $(n=6)$. By the day of LMAN lesions, global similarity $\mathrm{GS}_{2}$, and each of the component scores [percentage similarity (\%Sim), accuracy (Acc)] were significantly lower than their predeaf baseline. Data shown are group means $\pm \mathrm{SEM},{ }^{*} p<0.05$ (deaf-7 vs L0).

in all but one animal. The average volume of LMAN in lesioned animals was $32 \pm 8 \%$ of control LMAN volume. In the animals that retained a recognizable motif structure up to the time of LMAN lesion $(n=9), \mathrm{GS}_{1}$ scores improved rapidly after LMAN lesions (Fig. 4). In addition to there being a significant overall effect of the lesion $(F=19.38, n=7, p<0.005)$, post hoc comparisons confirmed that $\mathrm{GS}_{1}$ scores on L3 were significantly greater than on L0 $(p<0.05)$. This improvement in the group average remained significant through L28 $(p<0.05)$. In most animals, behavioral recovery was apparent as soon as song behavior commenced after lesion surgery (Fig. 5); within-animal analyses revealed that in five of eight birds that sang $1 \mathrm{~d}$ after LMAN lesion, the $\mathrm{GS}_{1}$ scores were already significantly greater than their L0 value $(p<0.05)$. The group average $\mathrm{GS}_{1}$ score reached a peak at $\mathrm{L} 7$, and analyses within animals revealed that scores on $\mathrm{L} 7$ were significantly greater than on $\mathrm{L} 0$ in seven of nine birds $(p<0.05)$. In contrast, significantly fewer birds (one of nine) increased their $\mathrm{GS}_{1}$ score in the week before lesion $\left(\chi^{2}=40.5 ; p<0.0001\right)$. Examples of the nature of behavioral deterioration after deafening and effects of LMAN lesion are shown in Figure 6.

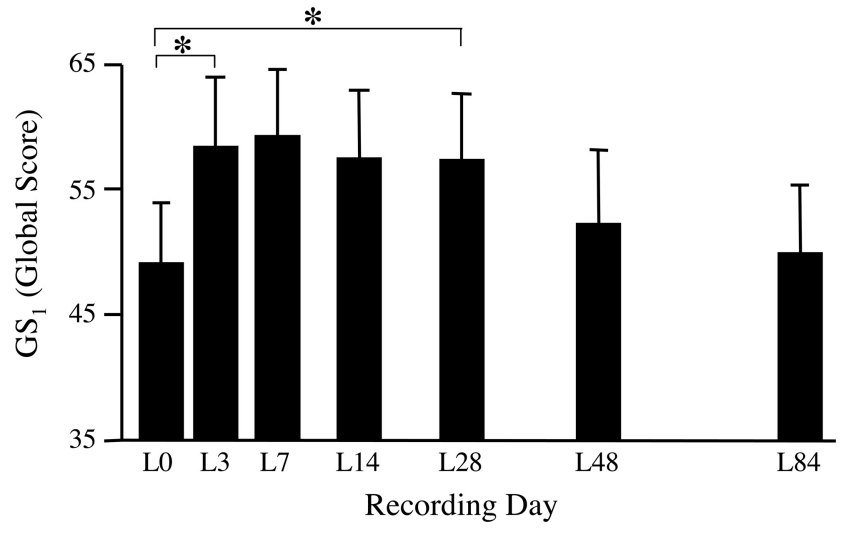

Figure 4. Bilateral LMAN lesions improved the global similarity score for birds that maintained song motif structure. Scores on L3 were significantly elevated above L0 scores, and this improvement was maintained through L28. Data shown are group means \pm SEM, ${ }^{*} p<0.05$.

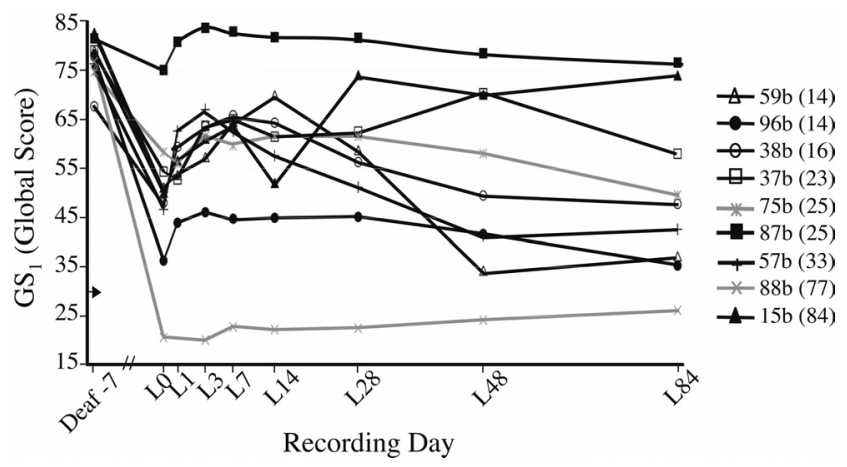

Figure 5. Global similarity scores for birds that maintained motif structure. Scores on L7 were significantly higher than on L0 for all birds except 88b and 75b (gray lines/symbols). The number of days elapsed between deafening and LMAN lesion is shown parenthetically in the legend. Arrow on $y$-axis indicates maximum score generated from comparison of predeaf motifs from unrelated males. Data shown are daily average scores for each animal.

In birds that retained motif structure, LMAN lesions also improved each of the individual measures that contribute to the $\mathrm{GS}_{1}$ score $(F \geq 14.72, p \leq 0.03)$ (supplemental Figs. $1-3$, available at www.jneurosci.org as supplemental material). Although the group average for $\mathrm{GS}_{1}$ peaked at $\mathrm{L}$, percentage similarity scores peaked at L3, by which time they were significantly elevated above L0 scores $(p<0.05)$. Both accuracy and sequential match scores peaked at $\mathrm{L} 7$, by which time they were significantly elevated above L0 scores $(p<0.01)$. Analyses within individual birds confirmed that LMAN lesions improved each of these measures in most birds. Of the seven birds that significantly improved $\mathrm{GS}_{1}$ after lesion, six also exhibited significant improvement in each of percentage similarity, accuracy, and sequential match (L7 vs L0, $p<0.05$ ). The seventh bird (96b) did not improve percentage similarity after lesion, but both accuracy and sequential match scores were significantly higher on L7 than on L0 $(p<0.001)$. For the two birds in the motif analysis whose $\mathrm{GS}_{1}$ did not improve significantly after LMAN lesion, significant improvement by L7 was evident only in the accuracy score for one bird $(p<0.001)$ and only in the sequential match score for the other bird $(p<0.001)$.

\section{LMAN lesions do not reverse severe vocal deterioration}

As described above, seven of nine birds that retained motif structure significantly improved similarity scores after LMAN lesions. Further analyses suggested that LMAN lesions recovered a greater proportion of the deafening-induce decline in birds with mild- 

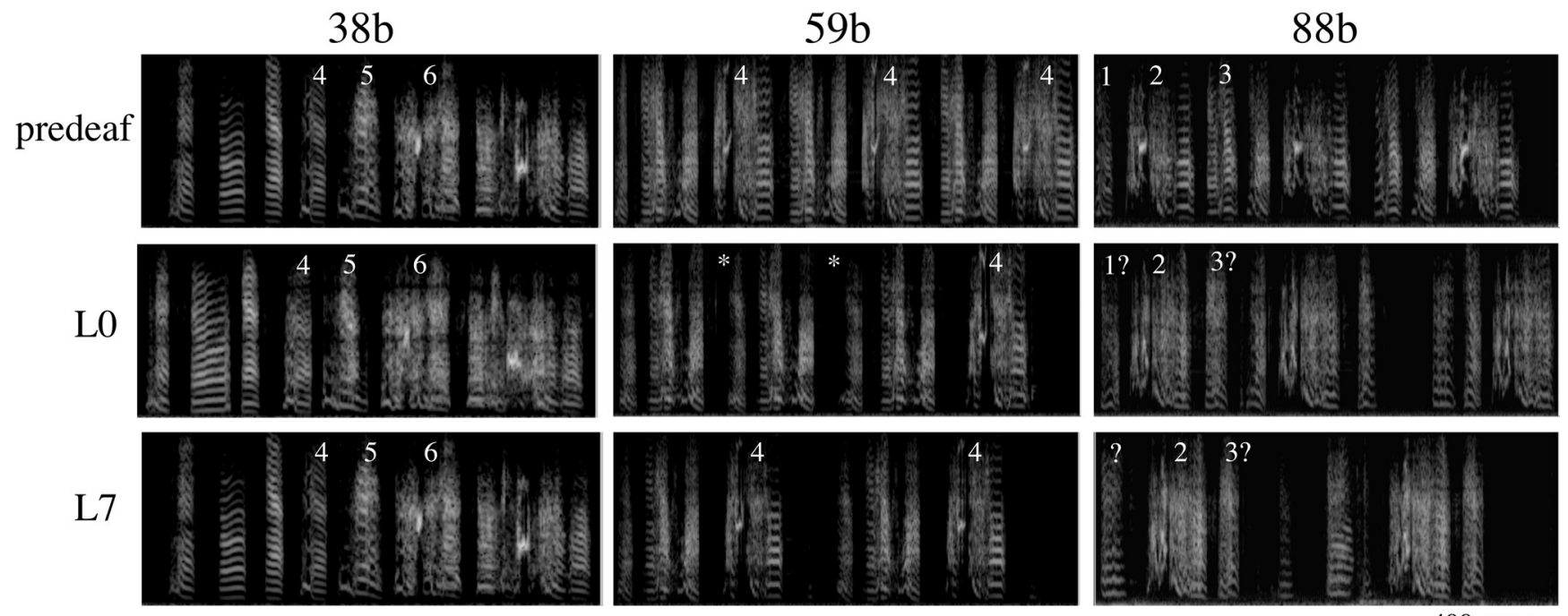

$400 \mathrm{msec}$

Figure 6. Sonograms of three representative birds (38b, 59b, $88 \mathrm{~b}$ ) recorded before deafening (top), just before LMAN lesion (middle), and $7 \mathrm{~d}$ after LMAN lesion. The bird on the left exhibited deafening-induced deterioration of syllable morphology (e.g., syllable nos. 4, 5, 6) that recovered after LMAN lesion. Particularly obvious is that LMAN lesions reversed deafening-induced changes in the periodicity of harmonically structured sounds (e.g., syllable 4). The bird in the middle frequently omitted syllable no. 4 in L0 songs (*) and LMAN lesion improved consistency in the production of this syllable. The bird on the right had severely degraded song by L0, and LMAN lesions did not improve similarity scores.

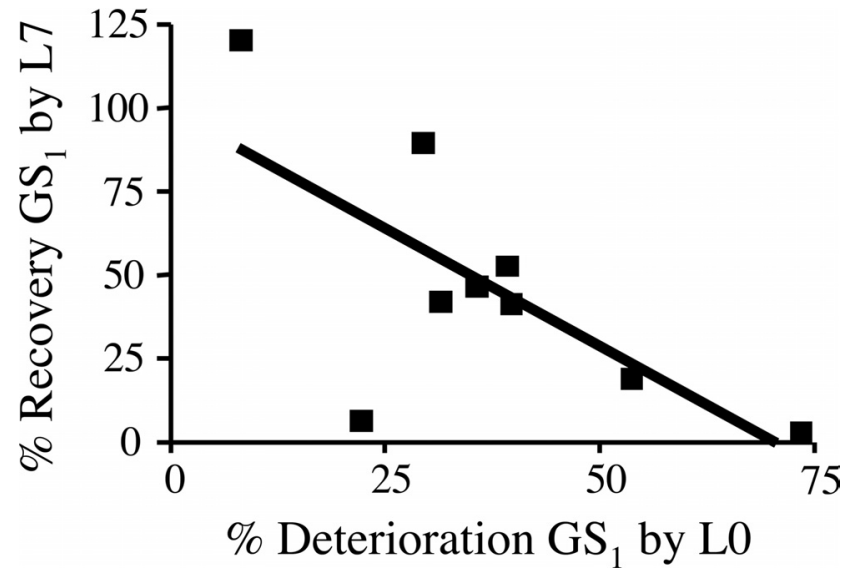

Figure 7. In deafened birds that maintained motif structure, the extent to which predeaf global similarity scores were recovered after LMAN lesion related to the degree of song deterioration by the time of LMAN lesion $\left(r^{2}=0.46 ; p<0.05\right)$. Birds with moderate behavioral deficits recovered more of their predeaf song structure than did birds with more severely deteriorated songs.

moderate degradation than in birds with more severe degradation. Among the nine birds that maintained a recognizable motif, those with moderately deteriorated $\mathrm{GS}_{1}$ scores recovered a larger proportion of their predeaf song structure than did those with more severely deteriorated song $\left(r^{2}=0.46 ; p<0.05\right)$ (Fig. 7). While this could reflect a fixed amount of lesion-induced improvement across all of the birds (which would be a larger percentage of moderate than severe declines), this was not the case. For instance, two birds in this group had completely deleted a syllable by L0, and LMAN lesions did not recover those syllables, nor did they promote improvement in $\mathrm{GS}_{1}$ scores. Furthermore, the bird with the least amount of degradation completely recovered predeaf levels of similarity but only gained modestly in similarity points (presumably due to a ceiling effect). Finally, among the remaining six birds in this group, the raw value of the $\mathrm{GS}_{1}$ score increased the most in the bird with the least degradation and increased the least in the bird with the most degradation.
The inverse relationship between degree of song degradation and LMAN lesion-induced recovery was also supported by the analysis of birds with profound vocal deterioration as reflected in loss of motif structure. In this group of birds $(n=6)$, LMAN lesions not only failed to improve global similarity $\left(\mathrm{GS}_{2}\right)$ scores, but these scores declined between L0 and L7 and were significantly lower by L28 than on L0 $(\mathrm{L} 0=53.23 \pm 2.2 ; \mathrm{L} 7=48.61 \pm$ 2.7; L28 $=45.08 \pm 3.1 ; p<0.01)$. Analyses of individual birds also indicated that LMAN lesions failed to promote recovery in these birds. In fact, in most birds that lost motif structure, $\mathrm{GS}_{2}$ scores continued to decline after LMAN lesion (Fig. 8, black symbols/lines). To evaluate whether this "long sample" analysis (see Materials and Methods) was robust enough to detect improvement in song similarity, we applied it to two birds in which LMAN lesions recovered $\mathrm{GS}_{1}$ scores measured by the motif analysis. In both of these birds, lesion-induced improvement in $\mathrm{GS}_{2}$ score was confirmed (Fig. 8, gray symbols) (L0 vs L7; $t>2.9, p<$ $0.01)$. When all birds were grouped according to whether or not they exhibited significant recovery, groups did not differ significantly in age at deafening (132 \pm 3 vs $132 \pm 2)$, days elapsed between deafening and LMAN lesion $(30 \pm 9$ vs $47 \pm 12)$ or volume of LMAN spared (percentage of control volume, $40 \pm$ $14 \%$ vs $24 \pm 7 \%)$.

\section{LMAN lesion-induced recovery of motif consistency}

LMAN lesions not only improved similarity scores, they also reduced the variability of songs produced after deafening (Fig. 9). In the seven birds that individually showed significant recovery of $\mathrm{GS}_{1}$ scores after lesion (henceforth referred to as "recovered" group), the $\mathrm{CV}$ of $\mathrm{GS}_{1}$ increased significantly after deafening (d-7 vs L0: $W=28, p<0.02$ ), and LMAN lesions resulted in an overall reduction in this $\mathrm{CV}$ that approached statistical significance $(F=$ 12.24, $p=0.057)$. Post hoc $t$ tests revealed that the CV of $\mathrm{GS}_{1}$ scores was significantly lower on L3 than on L0 $(p<0.05)$, and this effect was still evident on L84 $(p<0.05)$. In this group of recovered animals, LMAN lesions significantly reduced the CV of percentage similarity and accuracy $(F \geq 18.86, p \leq 0.005$; data not shown), but the CV of sequential match scores was not obvi- 


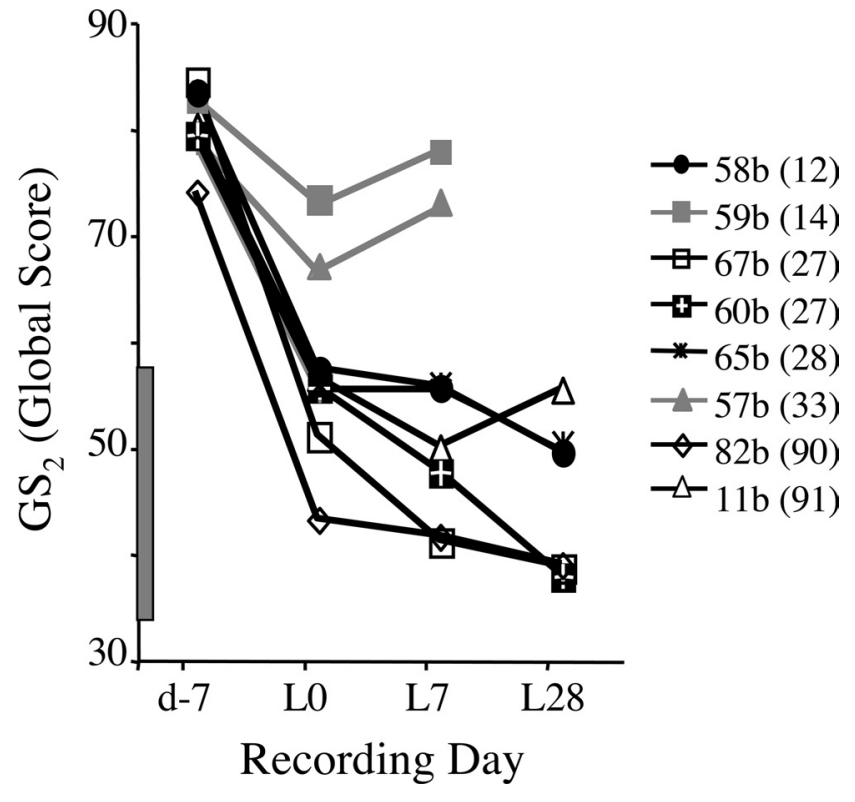

Figure 8. In deafened birds that lacked motif structure by LO ( $n=6$; black symbols), LMAN lesions did not improve $\mathrm{GS}_{2}$ scores. Also plotted are scores for two of the birds ( $\mathrm{db} 57 \mathrm{~b}$ and $\mathrm{db} 59 \mathrm{~b}$; gray symbols) shown in Figure 4 that were reanalyzed using the longer song sample analysis (see Materials and Methods). Songs of birds that lacked motif structure stabilized or continued to deteriorate after LMAN lesion, while significant behavioral recovery in the two birds that retained motif structure was confirmed by this analysis. Gray bar along $y$-axis indicates range of scores obtained from $\mathrm{L} 0$ samples of these same birds compared with predeaf templates from unrelated males. The number of days that elapsed between deafening and LMAN lesion is shown parenthetically in the legend. Data shown are daily average scores for each animal.

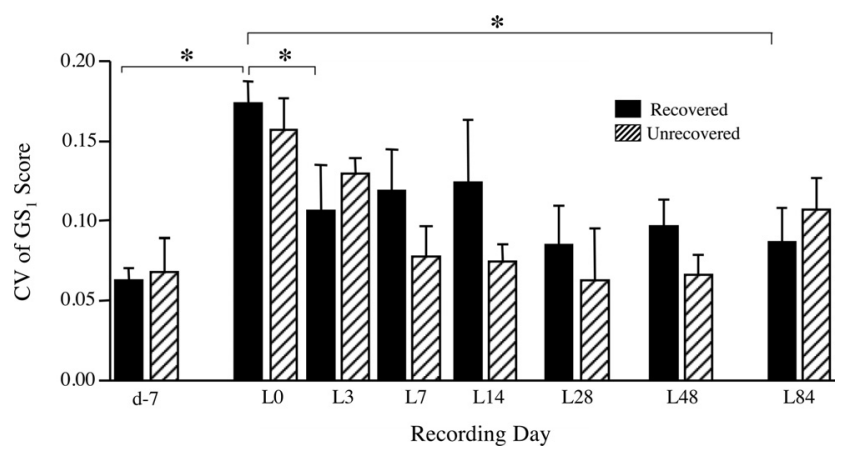

Figure 9. Adult deafening increased and bilateral LMAN lesions decreased variability across motifs (i.e., the $\mathrm{CV}$ of global similarity $\mathrm{GS}_{1}$ ) for birds that maintained song motif structure. For the recovered animals $(n=7)$, scores increased significantly after deafening ( $d-7$ vs $L 0: W=$ $\left.28,{ }^{*} p<0.02\right)$, and LMAN lesions resulted in an overall reduction in this (V that approached statistical significance $(F=12.24, p=0.057)$. Post hoct tests revealed that the V $_{\text {of }} \mathrm{SS}_{1}$ scores was significantly lower on $\mathrm{L} 3$ than on $\mathrm{LO}\left({ }^{*} p<0.05\right)$, and this effect was still evident on $\mathrm{L} 84$ $\left({ }^{*} p<0.05\right)$. Although unrecovered animals $(n=2)$ could not be evaluated statistically, they showed a similar pattern. Data shown are group means \pm SEM.

ously affected $(p=0.19)$. These effects of deafening and LMAN lesion on $\mathrm{CV}$ of the $\mathrm{GS}_{1}$ score were not restricted to animals that improved song similarity after LMAN lesions. Although only two animals that retained their motif structure did not improve $\mathrm{GS}_{1}$ after LMAN lesion, their average decline in CV after lesion was similar to that seen in the recovered birds (Fig. 9). Unfortunately, among the remaining "unrecovered" birds, absence of a motif structure precluded meaningful assessment of CV, because the long sample analysis permitted variable opportunities for similarity matches.
Although LMAN lesions could recover some aspects of song structure as described above, they did not restore a deafeninginduced decrease in the average number of motifs per song bout. Group analysis of all birds that retained a motif structure revealed that the average number of motifs per bout was significantly greater before deafening than on the morning of LMAN lesion $(3.5 \pm 0.4$ vs $2.3 \pm 0.3, t=3.07, p<0.05)$. By L7, the number of motifs per bout $(2.9 \pm 0.4)$ was not significantly different from the number just before LMAN lesion. Similarly, a within-bird analysis confirmed that each bird experienced a significant decrease in this temporal measure of song $(p<0.05)$, but none exhibited significant recovery by L7.

\section{Effects of deafening and LMAN lesions on syllable features and consistency}

Deafening affected five of eleven measures of syllable acoustic structure, and LMAN lesion reversed all but one of these effects (see Table 1). From d0 to L0, syllables from recovered ( 7 birds, 36 syllables) and unrecovered (4 birds, 17 syllables) groups increased mean entropy (noisiness) by $11 \%(W=-500, p<$ $0.0001)$ and $15 \%(W=-99, p<0.02)$, respectively. In the same time frame, syllables in recovered and unrecovered groups declined in pitch goodness (periodicity) by $12 \%$ ( $W=468, p \leq$ $0.0003)$ and $24 \%(W=143, p \leq 0.001)$ respectively. LMAN lesions did not significantly improve mean entropy in either subgroup, but it did improve mean pitch goodness in the recovered birds only ( $W=-450, p \leq 0.0005)$. Consistent with these results, close inspection of the frequency spectrograms revealed particularly obvious effects of LMAN lesion on the periodicity of harmonically structured sounds in recovered birds (Fig. 6). Deafening also affected some intrasyllable measures of feature variance (Table 1). By L0, entropy variance had declined (i.e., noisiness was less variable) from predeaf values by 29 and $53 \%$ in the recovered and unrecovered group, respectively ( $W \geq 125$, $p<0.005)$. Deafening also decreased variance of both pitch goodness and FM. In recovered and unrecovered groups, variance of pitch goodness declined from d0 to L0 by 16 and $26 \%$, respectively ( $W \geq 89, p<0.04$ ), and FM variance declined by 8 and $14 \%$, respectively ( $W \geq 111, p<0.01$ ). LMAN lesions reversed all of these effects, but in the recovered group only. The percentage of the deafening-induced decline that recovered by L7 was $\sim 50 \%$ for entropy variance $(W=-306, p<0.02), 100 \%$ for variance of pitch goodness ( $W=-440, p<0.001)$, and $73 \%$ for FM variance $(W=-344, p<0.01)$.

Although a previous report described a significant decline in average syllable duration after deafening (Brainard and Doupe, 2001) we did not observe such an effect. This discrepancy is likely due to methodological differences. Here, the same syllables were measured at each time point. In contrast, Brainard and Doupe measured the average duration of all syllables produced, and thus their results may reflect preferential loss of long syllables, additions of short syllables, increased repetition of short syllables, etc.

The most widespread effects of deafening and LMAN lesions on syllable features were observed in measures of their consistency across renditions. Among the subgroup of 36 syllables from recovered birds (Fig. 10), deafening significantly increased the CV of eight of eleven features: syllable duration, mean FM, mean pitch goodness, mean frequency, variation of FM, variation of mean pitch, variation of entropy, and variation of pitch goodness) ( $W \leq-280, p<0.03)$. Similar effects of deafening were evident among the subgroup of 17 syllables from the unrecovered group (supplemental Fig. 4, available at www.jneurosci.org as supplemental material) but reached statistical significance only 
Table 1. Means ( \pm SEM) of song syllable features that were affected by deafening and effects of LMAN lesion on those features

\begin{tabular}{|c|c|c|c|c|c|}
\hline & Entropy & Pitch goodness & Variation in entropy & Variation in pitch goodness & Variation in FM \\
\hline \multicolumn{6}{|c|}{ Recovered } \\
\hline do & $-1.86 \pm 0.10$ & $729 \pm 49$ & $0.54 \pm 0.08$ & $287,794 \pm 31,191$ & $610 \pm 27$ \\
\hline L0 & $-1.64 \pm 0.08^{*}$ & $639 \pm 41^{*}$ & $0.39 \pm 0.06^{*}$ & $242,937 \pm 24,898^{*}$ & $563 \pm 19^{*}$ \\
\hline L7 & $-1.66 \pm 0.08$ & $717 \pm 50^{\#}$ & $0.47 \pm 0.06^{\#}$ & $299,631 \pm 35,494^{\#}$ & $597 \pm 23^{\#}$ \\
\hline \multicolumn{6}{|c|}{ Unrecovered } \\
\hline do & $-1.53 \pm 0.11$ & $628 \pm 71$ & $0.59 \pm 0.09$ & $253,084 \pm 53,354$ & $608 \pm 35$ \\
\hline LO & $-1.29 \pm 0.09^{*}$ & $475 \pm 52^{*}$ & $0.27 \pm 0.04^{*}$ & $186,919 \pm 53,956^{*}$ & $522 \pm 26^{*}$ \\
\hline L7 & $-1.34 \pm 0.11$ & $498 \pm 58$ & $0.29 \pm 0.05$ & $183,782 \pm 48,252$ & $511 \pm 28$ \\
\hline
\end{tabular}

${ }^{a}$ Data are shown separately for birds in which global similarity scores improved after LMAN lesion (recovered; 7 birds, 36 syllables) and those that did not improve (unrecovered; 4 birds, 17 syllables). ${ }^{*} p<0.05$ (d0 versus L 0 ); $\# p<0.05$ (LO vs L7).

for the CV of syllable duration, mean frequency, variation of FM, and variation of pitch goodness ( $W \leq-97, p<0.03$;). The more limited statistical effects among this subgroup of animals may reflect the reduced power available in this relatively small sample size. In the recovered group, LMAN lesions reversed many of the deafening-induced increases in variability and even reduced the $\mathrm{CV}$ of some measures that were not affected significantly by deafening (Fig. 10). The CV was significantly lower on L7 than on L0 ( $W \geq 316$, $p \leq 0.02$ ) for all features of song syllables measured except mean entropy, mean frequency, variation of mean pitch, and variation of mean frequency. While declines in the CV of virtually all features were also observed after LMAN lesion in the unrecovered group (supplemental Fig. 4, available at www.jneurosci.org as supplemental material), differences between L0 and L7 were only statistically significant for the $\mathrm{CV}$ of mean frequency, variation of FM, variation of pitch goodness, and variation of mean frequency $(W \geq 93, p<0.03)$.

\section{Discussion}

The avian forebrain region LMAN conveys output from a basal ganglia-thalamic-pallial circuit to the principal vocal motor pathway and is required for the vocal experimentation and feedback-guided validation of vocal output that birds use to learn and maintain stable, stereotyped song patterns (Williams and Mehta, 1999; Kao et al., 2005; Olveczky et al., 2005; Kao and Brainard, 2006; Kao et al., 2008; Andalman and Fee, 2009). LMAN is also necessary for the initiation of adult vocal plasticity that normally occurs after deafening (Brainard and Doupe, 2000b); however, it has been unclear in this context whether LMAN is detecting discrepancies between actual and expected feedback, generating error signals in response to a mismatch, or actually participating in song modification (as in instances of adaptive vocal change). Here, we show that lesioning LMAN, even after deafening-induced changes have emerged, promotes rapid recovery toward the original song pattern. The results suggest that deafening-induced vocal degradation initially depends on the same processes that provoke adaptive vocal change. That is, LMAN directly participates in vocal degradation, alongside any role it plays in evaluating feedback or generating error signals. Additionally, we find that LMAN lesions are less effective in rescuing song structure once vocal degradation is severe, indicating Variation.

\section{Recovered}

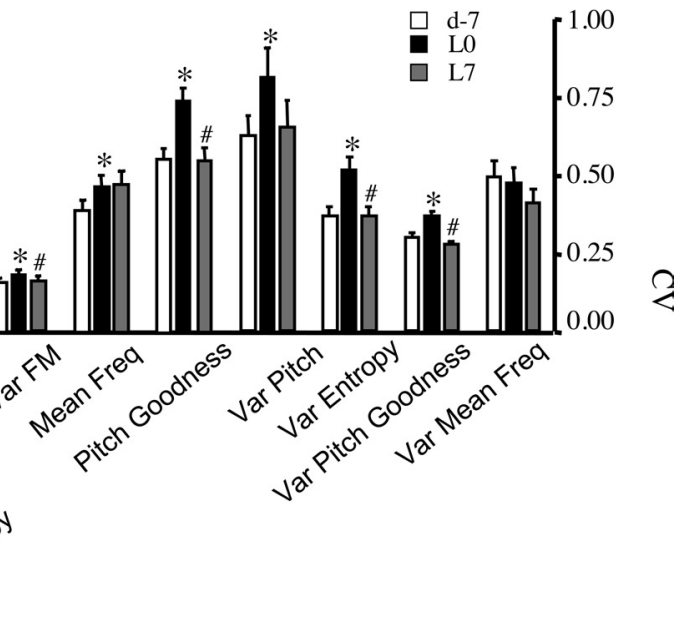

ne

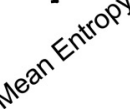
Figure 10. CV of syllable features for birds that exhibited significant recovery of GS $\mathrm{S}_{1}$ similarity scores after LMAN lesions (7 birds,
36 syllables). Adult deafening increased and bilateral LMAN lesions decreased variability across syllable renditions, as indicated by significant changes in the $\mathrm{CV}$ of most features. Data shown are group means $\pm \mathrm{SEM}$. The first seven features are plotted on the left axis, and the last four features are plotted on the right axis. ${ }^{*} p<0.05$ or ${ }^{\%} p=0.056$ for d0 vs L0, ${ }^{\#} p<0.05$ for LO vs L7. Var,

that behaviorally relevant neural changes ultimately occur outside of this region (e.g., within the VMP) to sustain vocal change. Since lesioning LMAN before deafening precludes severe song degradation, our results suggest that LMAN both contributes directly to the initial deafening-induced changes in song behavior and permits more enduring changes in other parts of the song system.

This is the first demonstration that LMAN contributes directly to the behavioral deterioration caused by deafening. However, LMAN lesions also rescue changes in song behavior caused by microlesions of HVC (Thompson et al., 2007), and while it is not clear whether the initial vocal deficits result from HVC damage, altered feedback, or both, questions concerning LMAN's role in behavioral recovery are similar in the two cases. Specifically, Thompson et al. argue that song degradation may reflect changes in how HVC and LMAN interact to influence vocal output. Damaging HVC could prompt behavioral degradation by immediately increasing LMAN's influence on RA neurons. Then, LMAN lesions would restore HVC's dominance over behavior and accelerate recovery. Similarly, deafening may gradually decrease the strength of HVC's output to RA, thereby increasing LMAN's influence over song. In this scenario, LMAN neurons need not actively alter their output in response to altered feedback, but simply gain more control over the VMP through decreased HVC activity. In fact, silencing HVC in adult, hearingintact birds induces a variable, unstructured song that is driven 
by LMAN, similar to subsong in younger birds (Aronov et al., 2008). Interestingly, perturbing auditory feedback in adult birds reduces HVC activity, which could increase LMAN's influence over behavior (Sakata and Brainard, 2008). Additionally, reduced HVC activity correlates with increased song variability, a relationship that could be mediated by the AFP (Day et al., 2008).

Deafening could also affect the interaction between RA inputs by increasing the strength and/or variability of LMAN neuronal activity, as in other instances of vocal plasticity (Kao et al., 2005, 2008; Olveczky et al., 2005; Kao and Brainard, 2006). While studies have failed to find evidence of immediate, feedback-induced changes in LMAN activity (Hessler and Doupe, 1999; Leonardo, 2004), tracheosyringeal nerve cuts promote gradual retuning of LMAN neurons, and this retuning predicts subsequent decrystallization of song (Roy and Mooney, 2007). Long-latency changes in LMAN activity after chronic deafening have not been examined. Perhaps such changes depend on alterations in a motorrelated, predictive corollary discharge (e.g., from HVC). If so, they would develop gradually since they would depend on sensorimotor remapping within upstream regions (Brainard and Doupe, 2000a).

Any change in LMAN or HVC activity resulting from a mismatch between expected and actual feedback is, in the broadest sense, an error signal. However, such a signal need not be "instructive"; that is, it need not bias motor exploration toward the intended target. Indeed, our results suggest that the neural changes produced by deafening cause song to move away from this target even though the motor template remains intact (as evidenced by the rapid recovery following LMAN lesions). Perhaps auditory feedback is needed for LMAN to provide instructive input to the motor pathway. In support of this idea, normal hearing adult birds can adaptively alter vocal output (Sober and Brainard, 2009), and this ability depends on LMAN (Andalman and Fee, 2009). Alternatively LMAN's role may be to provide random but purposive variation that serves as raw material for reinforcement-guided behavioral change. This could occur without LMAN needing to evaluate the nature of mismatches between feedback and stored song representations. In hearing birds, adaptive adjustments to song would emerge from both error-driven exploration and selective reinforcement (Doya and Sejnowski, 1994; Troyer and Bottjer, 2001). But in the absence of feedback, such exploration would be unguided and an otherwise intact motor memory would be obscured by error-driven changes in LMAN or HVC activity (Brainard and Doupe, 2000a). Finally, while there is considerable evidence from other paradigms that changes in LMAN activity directly cause behavioral changes similar to those reported here (e.g., increased variability), we cannot rule out the possibility that deafening-induced changes in song reflect changes in the activity of HVC-RA afferents or RA itself. In this case LMAN's role would be neuromodulatory, somehow allowing these changes to manifest themselves in behavior.

Understanding deafening-induced changes in song behavior as a product of interactions between HVC and/or LMAN activity has implications for understanding individual, age-related, and species differences in the response to adult deafening. For instance, individual differences in the rate of vocal deterioration reported here and previously (Nordeen and Nordeen, 1992; Brainard and Doupe, 2000b; Horita et al., 2008) may reflect natural variability in the degree to which HVC versus LMAN control RA output. The size of these structures (especially HVC) differs tremendously among individual adult zebra finches (Ward et al., 1998; Airey et al., 2000). Also, the diminishing effects of deafening with age (Lombardino and Nottebohm, 2000) could reflect a decline in the overall level of variability in LMAN activity (Kao and Brainard, 2006), and/or a greater influence of HVC-RA connections over behavior. Perhaps HVC-RA synapses become more stable over time, or the age-related decrease in the addition of new HVC neurons (Wang et al., 2002) reduces the susceptibility of the HVC-RA pathway to LMAN activity. In fact, if the incidence of new HVC-RA neurons impacts the VMP's response to LMAN activity, this also could account for species differences in the rate of deafening-induced song degradation (Scott et al., 2000).

Interestingly, LMAN lesions failed to promote recovery among birds exhibiting the most drastic deafening-induced changes. Thus, severe vocal deterioration likely reflects changes in the song system outside of LMAN, even though LMAN is necessary for development of these latter changes (Brainard and Doupe, 2000b). It seems likely that these more severe and enduring changes in song structure reflect alterations in the HVC-RA pathway, and LMAN clearly influences functional connectivity within this pathway during learning. In juvenile male zebra finches, LMAN lesions accelerate age-related changes in the density and strength of HVC-RA connections (Kittelberger and Mooney, 1999) and cause premature song crystallization (Bottjer et al., 1984; Scharff and Nottebohm, 1991). While it is not known whether similar AFP-regulated changes in HVC-RA connectivity accompany feedback-based adult vocal plasticity, some evidence is consistent with this hypothesis. For example, adult deafening induces molecular change within RA indicative of synaptic plasticity (Watanabe et al., 2002), and LMAN lesions prevent this molecular change (Watanabe et al., 2006).

Deafening-induced change in the HVC-RA pathway could develop as a result of the reversible changes initially driven directly by LMAN activity. For instance, while adaptive corrections in song produced by disruptive feedback are initially driven by LMAN, they are incorporated into the VMP within 1 day of learning (Andalman and Fee, 2009). Although the deafening-induced changes in song reported here take much longer to become independent of LMAN, this may reflect the absence of feedback necessary to evaluate motor exploration. LMAN also could encourage deafening-induced change in the HVC-RA pathway by providing essential trophic support to RA. LMAN neurons express and transport BDNF to RA (Johnson et al., 1997), and in adult birds BDNF infusions into RA increase both RA spine density and HVC terminal bouton frequency, while also causing changes in song behavior (Kittelberger and Mooney, 2005). In either case, it will be important to determine whether HVC-RA functional connectivity changes after adult deafening and whether the restorative effects of LMAN lesions diminish in relation to the development of such changes.

Based on its connections and pallial origin, LMAN may be functionally akin to prefrontal cortex (PFC) in mammals (Doupe and Kuhl, 1999; Reiner et al., 2004). Our results bolster this view insofar as PFC also has been linked to goal-directed behavioral adjustment in response to sensory feedback (Miller and Cohen, 2001). The song system may provide crucial insight into how such feedback alters activity within basal ganglia-thalamic cortical loops and shed light on the cognitive-motor functions of cortical-basal ganglia circuitry in general.

\section{References}

Airey DC, Castillo-Juarez H, Casella G, Pollak EJ, DeVoogd TJ (2000) Variation in the volume of zebra finch song control nuclei is heritable: developmental and evolutionary implications. Proc Biol Sci 267:2099-2104. Andalman AS, Fee MS (2009) A basal ganglia-forebrain circuit in the song- 
bird biases motor output to avoid vocal errors. Proc Natl Acad Sci U S A 106:12518-12523.

Aronov D, Andalman AS, Fee MS (2008) A specialized forebrain circuit for vocal babbling in the juvenile songbird. Science 320:630-634.

Bottjer SW, Miesner EA, Arnold AP (1984) Forebrain lesions disrupt development but not maintenance of song in passerine birds. Science 224:901-903.

Brainard MS, Doupe AJ (2000a) Auditory feedback in learning and maintenance of vocal behaviour. Nat Rev Neurosci 1:31-40.

Brainard MS, Doupe AJ (2000b) Interruption of a basal ganglia-forebrain circuit prevents plasticity of learned vocalizations. Nature 404:762-766.

Brainard MS, Doupe AJ (2001) Postlearning consolidation of birdsong: stabilizing effects of age and anterior forebrain lesions. J Neurosci 21: 2501-2517.

Cowie R, Douglas-Cowie E (1992) Postlingually acquired deafness: speech deterioration and the wider consequences. Berlin: Mouton de Gruyter.

Day NF, Kinnischtzke AK, Adam M, Nick TA (2008) Top-down regulation of plasticity in the birdsong system: "premotor" activity in the nucleus HVC predicts song variability better than it predicts song features. J Neurophysiol 100:2956-2965.

Doupe AJ, Kuhl PK (1999) Birdsong and human speech: common themes and mechanisms. Annu Rev Neurosci 22:567-631.

Doya K, Sejnowski TJ (1994) A novel reinforcement model of birdsong vocalization learning. In: Advances in neural information processing systems (Tesauro G, Touretzky D, Leen T, eds), pp 101-108. Cambridge, MA: MIT.

Hampton CM, Sakata JT, Brainard MS (2009) An avian basal gangliaforebrain circuit contributes differentially to syllable versus sequence variability of adult Bengalese finch song. J Neurophysiol 101:3235-3245.

Hessler NA, Doupe AJ (1999) Singing-related neural activity in a dorsal forebrain-basal ganglia circuit of adult zebra finches. J Neurosci 19:10461-10481.

Horita H, Wada K, Jarvis ED (2008) Early onset of deafening-induced song deterioration and differential requirements of the pallial-basal ganglia vocal pathway. Eur J Neurosci 28:2519-2532.

Johnson F, Hohmann SE, DiStefano PS, Bottjer SW (1997) Neurotrophins suppress apoptosis induced by deafferentation of an avian motor-cortical region. J Neurosci 17:2101-2111.

Kao MH, Brainard MS (2006) Lesions of an avian basal ganglia circuit prevent context-dependent changes to song variability. J Neurophysiol 96:1441-1455.

Kao MH, Doupe AJ, Brainard MS (2005) Contributions of an avian basal ganglia-forebrain circuit to real-time modulation of song. Nature 433:638-643.

Kao MH, Wright BD, Doupe AJ (2008) Neurons in a forebrain nucleus required for vocal plasticity rapidly switch between precise firing and variable bursting depending on social context. J Neurosci 28:1323213247.

Kittelberger JM, Mooney R (1999) Lesions of an avian forebrain nucleus that disrupt song development alter synaptic connectivity and transmission in the vocal premotor pathway. J Neurosci 19:9385-9398.

Kittelberger JM, Mooney R (2005) Acute injections of brain-derived neurotrophic factor in a vocal premotor nucleus reversibly disrupt adult birdsong stability and trigger syllable deletion. J Neurobiol 62:406-424.

Leonardo A (2004) Experimental test of the birdsong error-correction model. Proc Natl Acad Sci U S A 101:16935-16940.

Lombardino AJ, Nottebohm F (2000) Age at deafening affects the stability of learned song in adult male zebra finches. J Neurosci 20:5054-5064.

Marler P, Peters S (1982) Developmental overproduction and selective attrition: new processes in the epigenesis of birdsong. Dev Psychobiol 15:369-378.

Miller EK, Cohen JD (2001) An integrative theory of prefrontal cortex function. Annu Rev Neurosci 24:167-202.

Nordeen KW, Nordeen EJ (1992) Auditory feedback is necessary for the maintenance of stereotyped song in adult zebra finches. Behav Neural Biol 57:58-66.

Okanoya K, Yamaguchi A (1997) Adult Bengalese finches (Lonchura striata var. domestica) require real-time auditory feedback to produce normal song syntax. J Neurobiol 33:343-356.

Olveczky BP, Andalman AS, Fee MS (2005) Vocal experimentation in the juvenile songbird requires a basal ganglia circuit. PLoS Biol 3:e153.

Reiner A, Perkel DJ, Bruce LL, Butler AB, Csillag A, Kuenzel W, Medina L, Paxinos G, Shimizu T, Striedter G, Wild M, Ball GF, Durand S, Gunturkun O, Lee DW, Mello CV, Powers A, White SA, Hough G, Kubikova et al. (2004) Revised nomenclature for avian telencephalon and some related brainstem nuclei. J Comp Neurol 473:377-414.

Roy A, Mooney R (2007) Auditory plasticity in a basal ganglia-forebrain pathway during decrystallization of adult birdsong. J Neurosci 27:63746387.

Sakata JT, Brainard MS (2008) Online contributions of auditory feedback to neural activity in avian song control circuitry. J Neurosci 28:1137811390.

Scharff C, Nottebohm F (1991) A comparative study of the behavioral deficits following lesions of various parts of the zebra finch song system: implications for vocal learning. J Neurosci 11:2896-2913.

Scott LL, Nordeen EJ, Nordeen KW (2000) The relationship between rates of HVc neuron addition and vocal plasticity in adult songbirds. J Neurobiol 43:79-88.

Scott LL, Nordeen EJ, Nordeen KW (2007) IMAN lesions prevent song degradation after deafening without reducing HVC neuron addition. Dev Neurobiol 67:1407-1418.

Sober SJ, Brainard MS (2009) Adult birdsong is actively maintained by error correction. Nat Neurosci 12:927-931.

Tchernichovski O, Nottebohm F, Ho CE, Pesaran B, Mitra PP (2000) A procedure for an automated measurement of song similarity. Anim Behav 59:1167-1176.

Tchernichovski O, Mitra PP, Lints T, Nottebohm F (2001) Dynamics of the vocal imitation process: how a zebra finch learns its song. Science 291:2564-2569.

Thompson JA, Wu W, Bertram R, Johnson F (2007) Auditory-dependent vocal recovery in adult male zebra finches is facilitated by lesion of a forebrain pathway that includes the basal ganglia. J Neurosci 27:1230812320.

Tramontin AD, Smith GT, Breuner CW, Brenowitz EA (1998) Seasonal plasticity and sexual dimorphism in the avian song control system: stereological measurement of neuron density and number. J Comp Neurol 396:186-192.

Troyer TW, Bottjer SW (2001) Birdsong: models and mechanisms. Curr Opin Neurobiol 11:721-726.

Waldenstein RS (1990) Effects of postlingual deafness on speech production: Implications for the role of auditory feedback. J Acoust Soc Am 88:2099-2114

Wang N, Hurley P, Pytte C, Kirn JR (2002) Vocal control neuron incorporation decreases with age in the adult zebra finch. J Neurosci 22:10864-10870.

Ward BC, Nordeen EJ, Nordeen KW (1998) Individual variation in neuron number predicts differences in the propensity for avian vocal imitation. Proc Natl Acad Sci U S A 95:1277-1282.

Watanabe A, Kimura T, Sakaguchi H (2002) Expression of protein kinase C in song control nuclei of deafened adult male Bengalese finches. Neuroreport 13:127-132.

Watanabe A, Li R, Kimura T, Sakaguchi H (2006) Lesions of an avian forebrain nucleus prevent changes in protein kinase $\mathrm{C}$ levels associated with deafening-induced vocal plasticity in adult songbirds. Eur J Neurosci 23:2447-2457.

Williams H, Mehta N (1999) Changes in adult zebra finch song require a forebrain nucleus that is not necessary for song production. J Neurobiol 39:14-28. 